\title{
Les formations à blocs de l'Obac de Sispony (Andorre) Essai d'interprétation
}

\section{The Obac de Sistony boulder formations}

\author{
J.-Ph. Desfarge \\ Centre de géographie physique H. Elhai - U.E.R. de Géographie-UPX Nanterre \\ Institut d'Estudis Andorran, Perpignan
}

Le versant méridional de la vallée du Riu Montaner, dans l'ouest de l'Andorre, présente des modelés très remarquables par leur singularité dans cette partie des Pyrénées. Le versant, dont la crête culmine à $2334 \mathrm{~m}$ au pic de Carroi, est échancré par cinq cirques. De chacun sort une langue de blocailles. De plus près le modelé est complexe, il juxtapose des formations à blocs physionomiquement très différentes.

La complexité et le télescopage des formes sur l'Obac de Sispony pourraient apporter des connaissances nouvelles à l'histoire de l'évolution quaternaire de la région, en particulier l'importance des héritages anciens.
Un travail très détaillé sur le terrain a porté sur un cirque : le Clot del Gel.

L'Obac de Sispony présente une dénivelée qui varie de $500 \mathrm{~m}$ à l'amont de la vallée du Riu Montaner, à $850 \mathrm{~m}$ en aval. La crête qui sépare la Sola d'Enclar de l'Obac de Sispony se situe, sur l'étroite zone cornéenne, au contact des terrains cristallins et sédimentaires. Le versant est taillé dans des calcaires métamorphisés du Carbonifère (Fig. 2). Les terrains devoniens sous-jacents à la base du versant n'affleurent à aucun endroit. De cette situation de contact des calcaires carbonifères, il résulte une grande fragilisation de la roche qui se traduit par un diaclasage très dense.

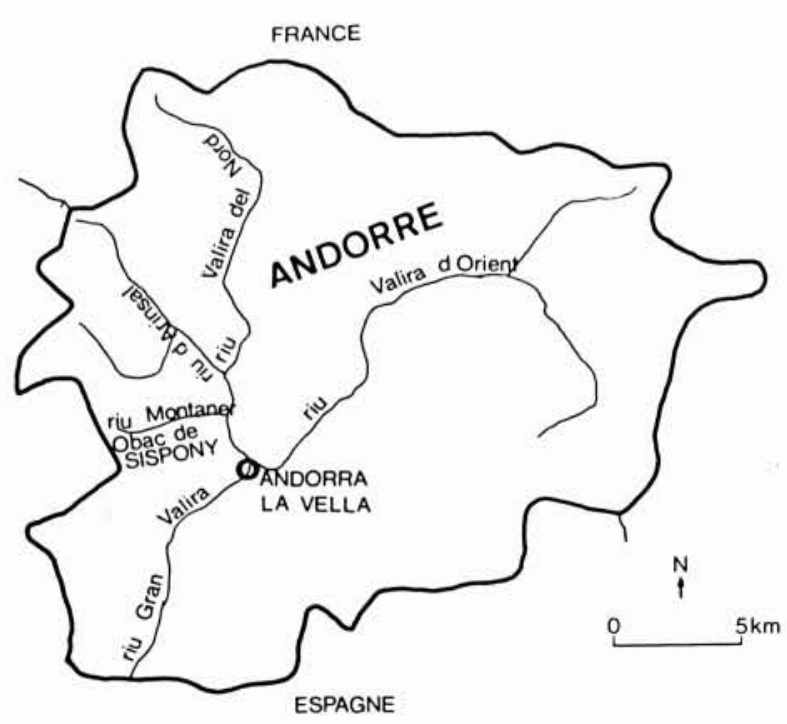

Figure 1 - Carte de localisation.

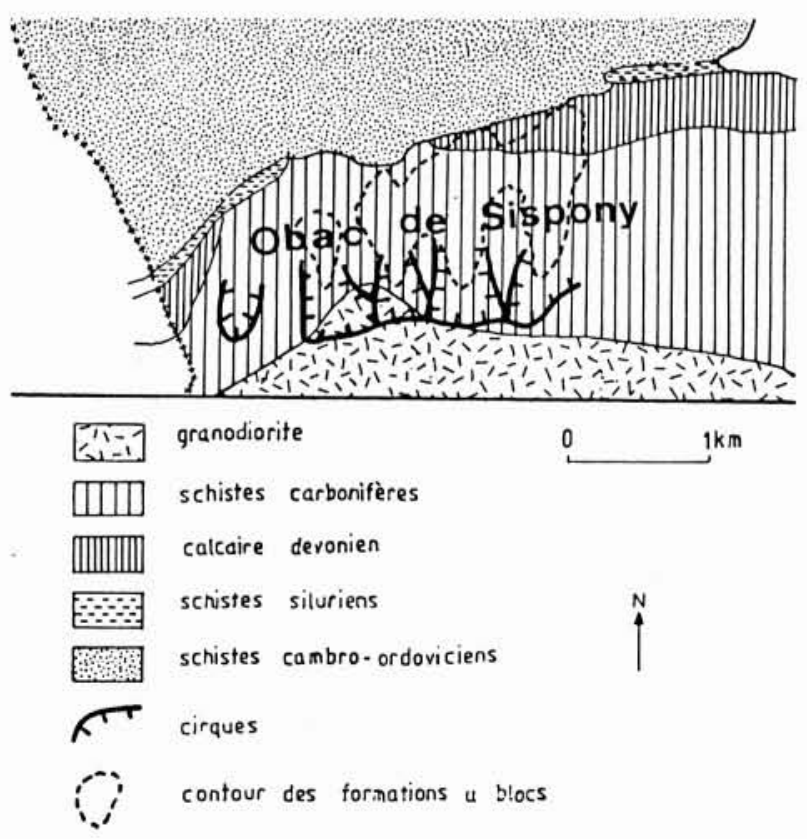

Figure $2-$ Croquis géologique de l'Obac de Sispony. 


\section{L'exemple du Clot del Gel (Fig. 3 et 4)}

Un géotransect effectué sur le Clot del Gel fait apparaître la succession suivante d'amont en aval (Fig. 3):

La paroi qui surplombe le cirque a un commandement de $200 \mathrm{~m}$ au maximum et une pente de $45^{\circ}$. Le diaclasage très serré des calcaires y a permis le développement de multiples ravins torrentiels, fonctionnant également en couloirs d'éboulis.

Le fond du cirque est, dans sa partie amont, remblayé par les éboulis qui descendent de la paroi. A l'aval de la paroi occidentale des traînées d'éboulis s'étalent jusqu'au débouché du cirque. La forme de ce dernier est fortement masquée par ce remblaiement; toutefois, il s'agit d'un entonnoir glaciaire élémentaire selon la classification de G. Galibert (1965).

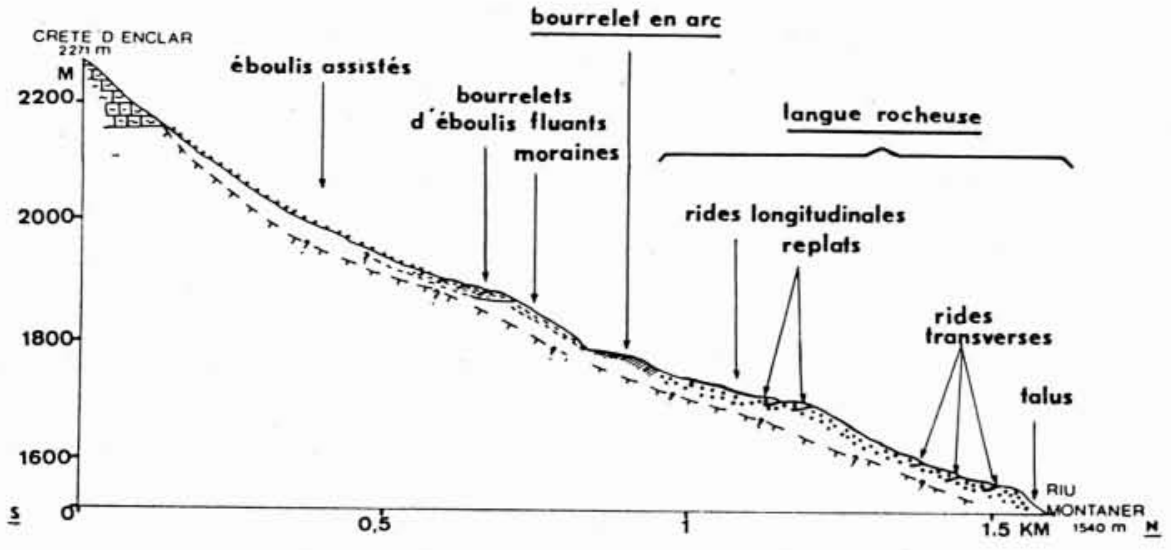

Figure 3 - Géotransect sur les formations à blocs de l'Obac de Sispony.

Vue d'ensemble des formations à blocs en aval du Clot del Gel.

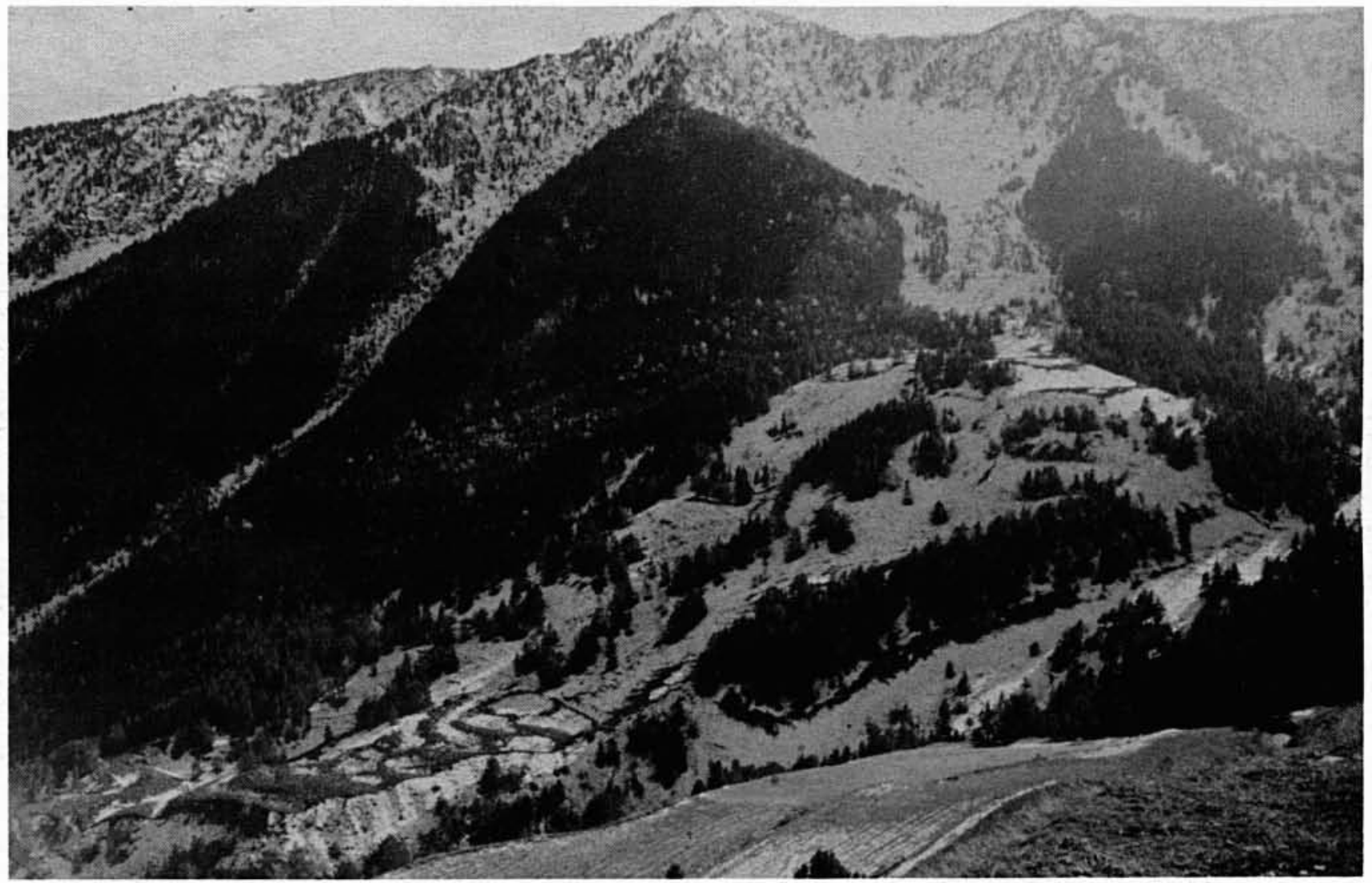


A l'extrémité aval du cirque, à $1860 \mathrm{~m}$ d'altitude, le débouché du cirque est ourlé de chaque côté par un bourrelet latéral qui semble se dédoubler à l'aval.

En contrebas de ce grand ensemble, la seconde unité physionomique est un bourrelet de forme en croissant, à l'altitude de $1760 \mathrm{~m}$. Une pente de $25^{\circ}$, longue de $200 \mathrm{~m}$ pour $80 \mathrm{~m}$ de dénivelée, le sépare de l'entrée du cirque. La pente interne du bourrelet est de $25^{\circ}$, la pente externe de $28^{\circ}$ et la hauteur relative de $4 \mathrm{~m}$. A l'amont, le croissant est limité par une dépression circulaire. Le matériau est relativement homométrique pour les blocs (une trentaine de $\mathrm{cm}$ en moyenne); la proportion de fines nulle en surface, devient rapidement importante en profondeur.

L'unité physionomique qui fait suite au bourrelet présente une beaucoup plus grande extension. Il s'agit d'une langue de blocs de calcaires primaires, de couleur brun-rouge. Dans l'ensemble, elle a l'aspect de celles déjà décrites par G. Angely (1967) dans les Pyrénées et V. Guiter (1972) dans les Alpes. Au sein de cette unité plusieurs morphoséquences bien différenciées sont distinguées. La moitié Ouest de la langue rocheuse est formée de rides longitudinales, alors qu'à l'Est les arcs sont transversaux.

La partie Est est formée d'une série de 4 arcs en forme de croissant aplati. Leur hauteur est de l'ordre de $1 \mathrm{~m}$ par rapport aux creux qui les séparent. Les blocs qui les composent sont de taille relativement homogène (pas de blocs de plus de $1 \mathrm{~m}$ de longueur). Cependant, les plus gros ont une localisation préférentielle sur les revers amont des bourrelets. La pente externe des arcs est de $30^{\circ}$. Les quatre bourrelets semblent plus encastrés les uns dans les autres que juxtaposés.

A l'Ouest, la pente longitudinale reste régulière $\left(10^{\circ}\right)$ pendant une quarantaine de mètres, mais dans la longueur, deux rides parallèles sont nettement dessinées. Leur hauteur relative par rapport au sillon est de l'ordre du mètre.

Transversalement, les rides peuvent être interrompues par de petits sillons, de $30 \mathrm{~cm}$ au maximum de profondeur. Deux replats font suite aux rides, ils n'ont plus l'allure, en profil, de croissant mais sont très aplatis.

En plan leur forme est semi-circulaire. Les talus et les creux qui les prolongent font chacun une trentaine de mètres de long, la pente des talus est de $25^{\circ}$ et la pente interne des replats d'une dizaine de degrés.

En-dessous de $1860 \mathrm{~m}$ la pente reste ensuite régulière pendant une cinquantaine de mètres, avec une inclinaison de $25^{\circ}$. Progressivement, elle devient ensuite irrégulière; vers $1630 \mathrm{~m}$, des replats d'extension limitée apparaissent.

Vers $1600 \mathrm{~m}$ d'altitude, commence le grand replat terminal.

La pente de cet ensemble basal se réduit considérablement jusqu'à devenir inférieure à $10^{\circ}$. Cinq rides peuvent être distinguées dans la partie occidentale. Leur hauteur relative par rapport aux sillons peut atteindre $2 \mathrm{~m}$. Dans la partie orientale, le télescopage des rides longitudinales et transverses rend la situation beaucoup plus complexe. Deux coupes ont été ouvertes dans les rides, elles montrent une relative homométrie des blocs, ceux supérieurs à $1 \mathrm{~m}$ sont rares. Ils sont disposés parallèlement à la pente. Plusieurs relevés de surface ont montré que l'orientation
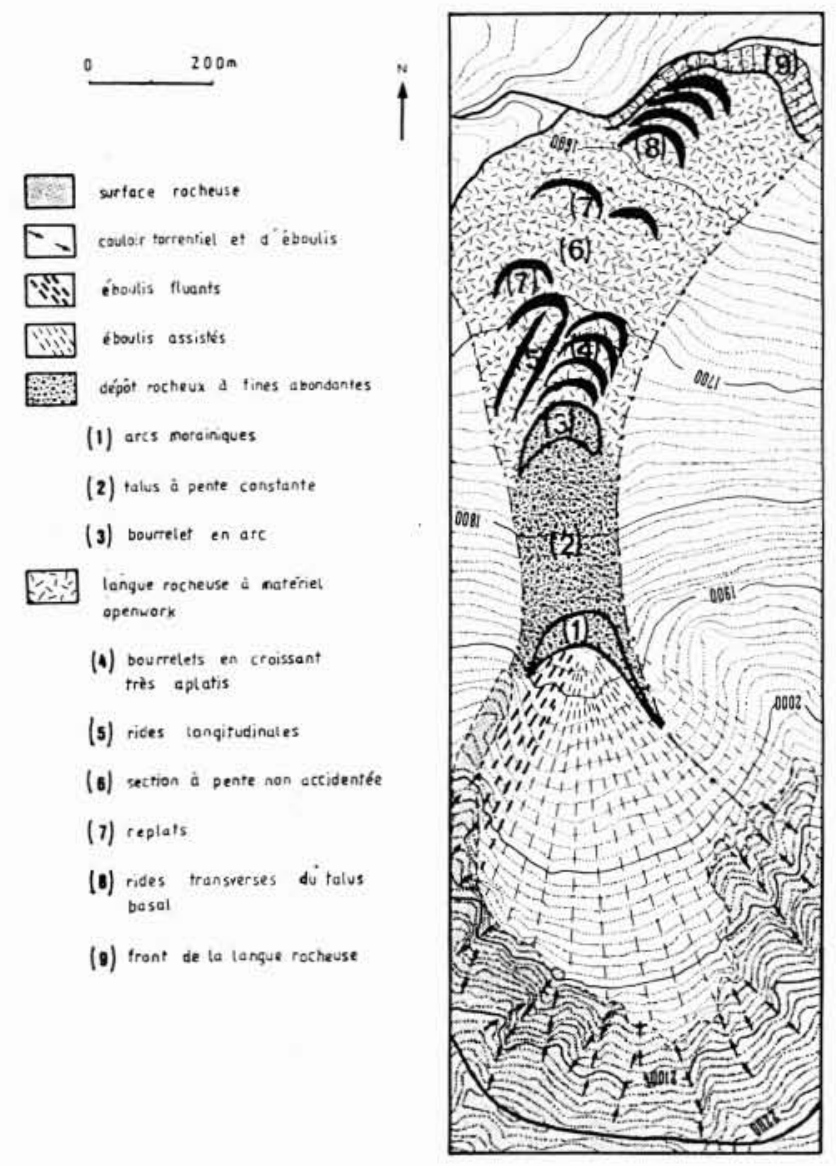

Figure 4 - Croquis géomorphologique du Clot del Gel.

du plus grand axe, dans la direction de la plus grande pente, est dominante.

La langue rocheuse se termine ensuite à l'aval par un talus en pente très forte (jusqu'à $42^{\circ}$ ) où les blocs relèvent nettement du nez vers l'aval. Le talus est remanié par éboulisation en certains points, comme le montre le granoclassement longitudinal.

Le géotransect sur l'Obac de Sispony montre, d'amont en aval :

- des formations d'éboulis associées aux moraines qui se localisent dans le cirque:

- un bourrelet dont on ne peut préciser, dans l'état actuel de nos recherches, s'il s'agit d'un bourrelet de névé ou d'un croissant morainique terminal;

- une langue rocheuses dont la topographie de surface dénote un modelage par un mouvement fluidal. Cette physionomie pourrait s'apparenter à celle des dépôts de glaciers rocheux. Cependant, la très faible altitude à laquelle se trouve cette formation oblige à s'en tenir au constat d'une convergence physionomique. 


\section{Le modelé du versant}

La description que nous donnons des grandes formes qui se développent sur l'Obac de Sispony est le résultat d'une première étude en photo-interprétation, la reconnaissance sur le terrain n'a pas encore été faite. Ceci explique le caractère incomplet des observations, car certaines formations à blocs sont recouvertes par la forêt. Un examen complémentaire sur le terrain est indispensable poùr isoler précisément les caračtères communs ou particuliers à chaque Clot.

A première vue, les formes développées à la surface de l'Obac de Sispony paraissent assez semblables, cependant les différences morphologiques sont sensibles. Les deux cirques occidentaux sont orientés à $\mathrm{N} 345^{\circ}$, le cirque oriental à $\mathrm{N} 25^{\circ}$, tandis que les deux autres sont plein Nord (Fig. 5).

- le modelé du Clot de la Sella ressemble à celui du Clot del Gel. Le bourrelet en arc se trouve ici à $1750 \mathrm{~m}$ d'altitude. Les moraines d'amont sont à $1860 \mathrm{~m}$. Là aussi, la langue rocheuse s'étire jusqu'au thalweg mais les formes de détail sont masquées par la couverture forestière;

- à l'Est, le Clot de la Rectoria possède un modelé singulier. Du cirque au bourrelet en arc les formes sont celles des autres Clots mais à partir de l'altitude $1700 \mathrm{~m}$ la langue pierreuse prend l'allure d'un vaste talus de pente régulière, aussi bien longitudinalement que transversalement. Il n'y a aucun granoclassement longitudinal du matériel dans cet ensemble;
- le modelé des Clots de la Coma del Pou et de la Coma del Prat comprend, comme pour les autres, des moraines vers $1880-1900 \mathrm{~m}$ d'altitude. En aval, les formes sont beaucoup moins nettes, le versant est ici beaucoup moins long et toutes les accumulations se sont télescopées. L'arrangement d'ensemble est donc difficilement perceptible. Tous ces modelés se trouvent également sous forêt.

On retrouve sur tout l'Obac de Sispony la même trilogie : moraines au débouché des cirques, bourrelet en arc à mi-hauteur et accumulation rocheuse en aval jusqu'au thalweg.

\section{La morphologie de l'Obac de Sispony replacée dans le contexte quaternaire de l'Ouest de l'Andorre}

Le travail, très approfondi, fait par M.C. Prat (1980) sous la direction de $\mathrm{P}$. Barrère, peut servir de base à la reconstitution du contexte quaternaire dans cette partie de l'Andorre (Fig. 6).

Lors de la période du Maximum, un glacier de vallée, épais de $300 \mathrm{~m}$, descendait des hauteurs du Massif de Coma Pedrosa, par la vallée du Riu d'Arinsal, pour entrer en confluence avec le glacier d'Ordino, $2 \mathrm{~km}$ en aval de la Massana. Cet ensemble rejoignait ensuite le glacier de la vallée du Valira d'Orient, dans le bassin d'Andorre la Vieille.
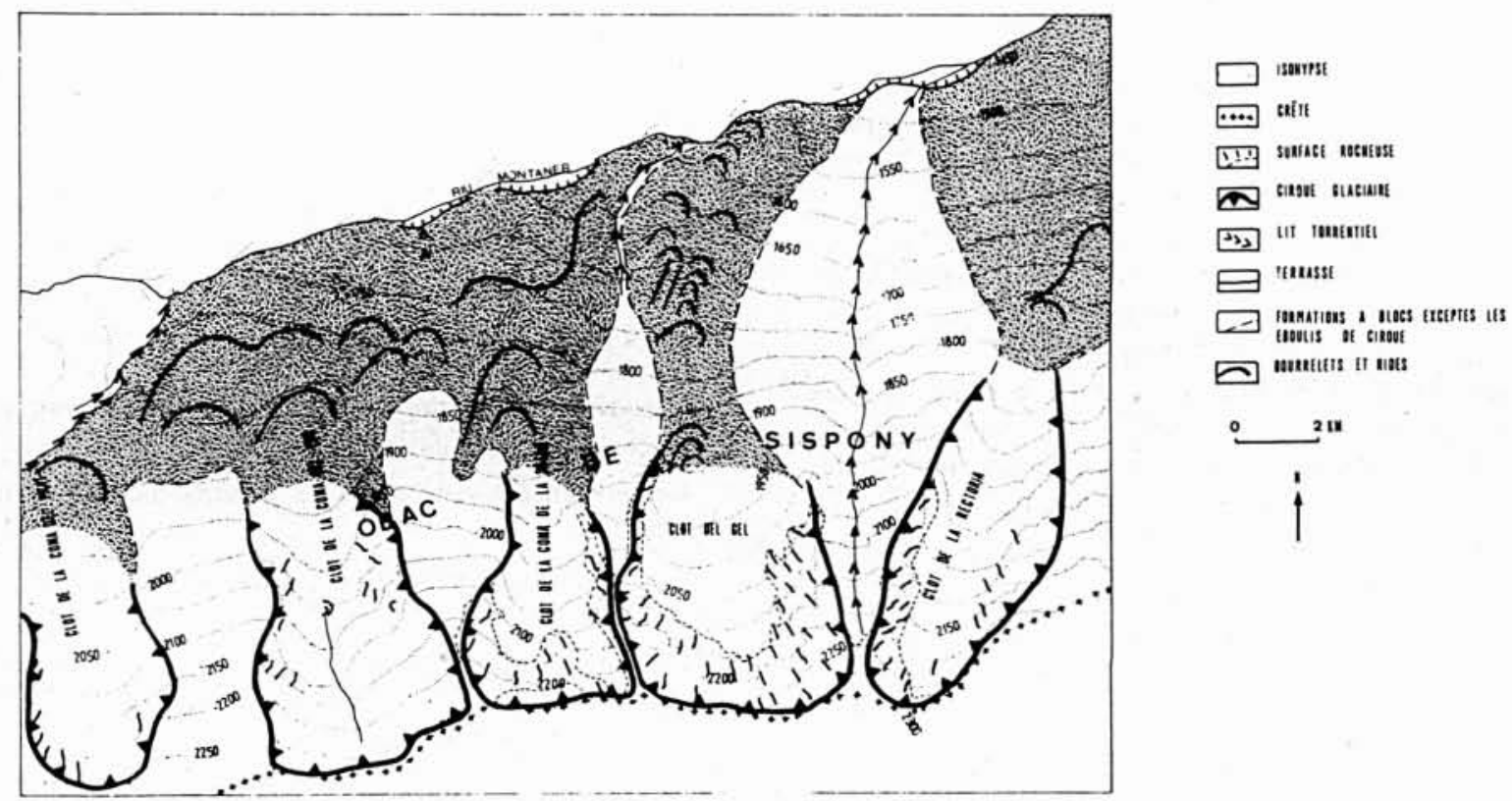

Figure 5 - Carte géomorphologique de l'Obac de Sispony. Premiers levés par photo-interprétation. 
A cette période, les vallées de Pal et du Riu Montaner n'ont pas été occupées par une langue de glace. Seuls quelques petits glaciers se sont logés dans de petits cirques sur les versants Nord et Est. Cette absence de glacier de vallée est imputable à la faible altitude des crêtes encadrantes. La crête de l'Obac de Sispony est d'ailleurs une des crêtes les plus basses des Pyrénées andorranes qui porte une empreinte glaciaire.

M.C. Prat attribue les formations à blocs de l'Obac de Sispony à la période du Maximum, son argumentation repose sur la puissance du dépôt. On peut ajouter que cette période a été effectivement la plus froide de l'histoire des glaciations pyrénéennes et qu'il apparait donc logique que les dépôts du versant lui soient attribués.

Dans ce schéma le bourrelet en arc est, pour M.C. Prat, daté de la phase de stationnement du Maximum glaciaire, alors que la formation rocheuse à l'aval est une moraine de la phase d'expansion.

Dans cette hypothèse, ces moraines, très anciennes, n'auraient pas subi de remaniement postérieur. Pourtant la fraicheur des formes à la surface de la langue rocheuse du Clot del Gel est surprenante : il n'y a pas de traces d'entailles de ruissellement et des pentes atteignant $40^{\circ}$ au front de la langue, se seraient maintenues malgré leur très grande ancienneté.

\section{La possibilité d'un remaniement en glacier rocheux au cours du Post-Maximum}

La possibilité de l'existence d'un glacier rocheux à une si faible altitude peut être avancée grâce aux recherches récentes menées dans les Alpes par M. Evin (1983) notamment. La comparaison de la limite d'activité des glaciers rocheux et de celles des neiges persistantes indique que les deux niveaux sont séparés par une dénivelée de $300 \mathrm{~m}$. Bien que les calculs aient été faits sur des moyennes régionales, ils gardent, cependant, une valeur indicative. Dans cette démarche il convient de signaler les réserves que requiert le maniement des deux limites et surtout celle des neiges persistantes, ainsi que la prudence qu'exige la projection dans le passé de limites relatives actuelles. Peu de recherches ont été faites dans les Pyrénées sur les rapports entre ces deux niveaux, toutefois F. Taillefer (1968) indique, pour le Néoglaciaire chronologiquement équivalent au Tardiglaciaire, une ligne d'équilibre des appareils glaciaires au niveau de $2600 \mathrm{~m}$ dans le Canigou, alors que les fronts de glaciers rocheux les plus éloignés se trouvent $600 \mathrm{~m}$ en contrebas (G. Soutadé, 1973).

En Andorre, pour la période du Post-Maximum, la limite d'équilibre glaciaire est fixée par M.C. Prat à $2300 \mathrm{~m}$. La limite d'activité des glaciers rocheux pourrait être $300 \mathrm{~m}$ en contrebas. Malgré toutes les incertitudes de cette méthode, les moraines du Maximum sur l'Obac de Sispony, ont donc pu connaître une dynamique de type glacier rocheux.

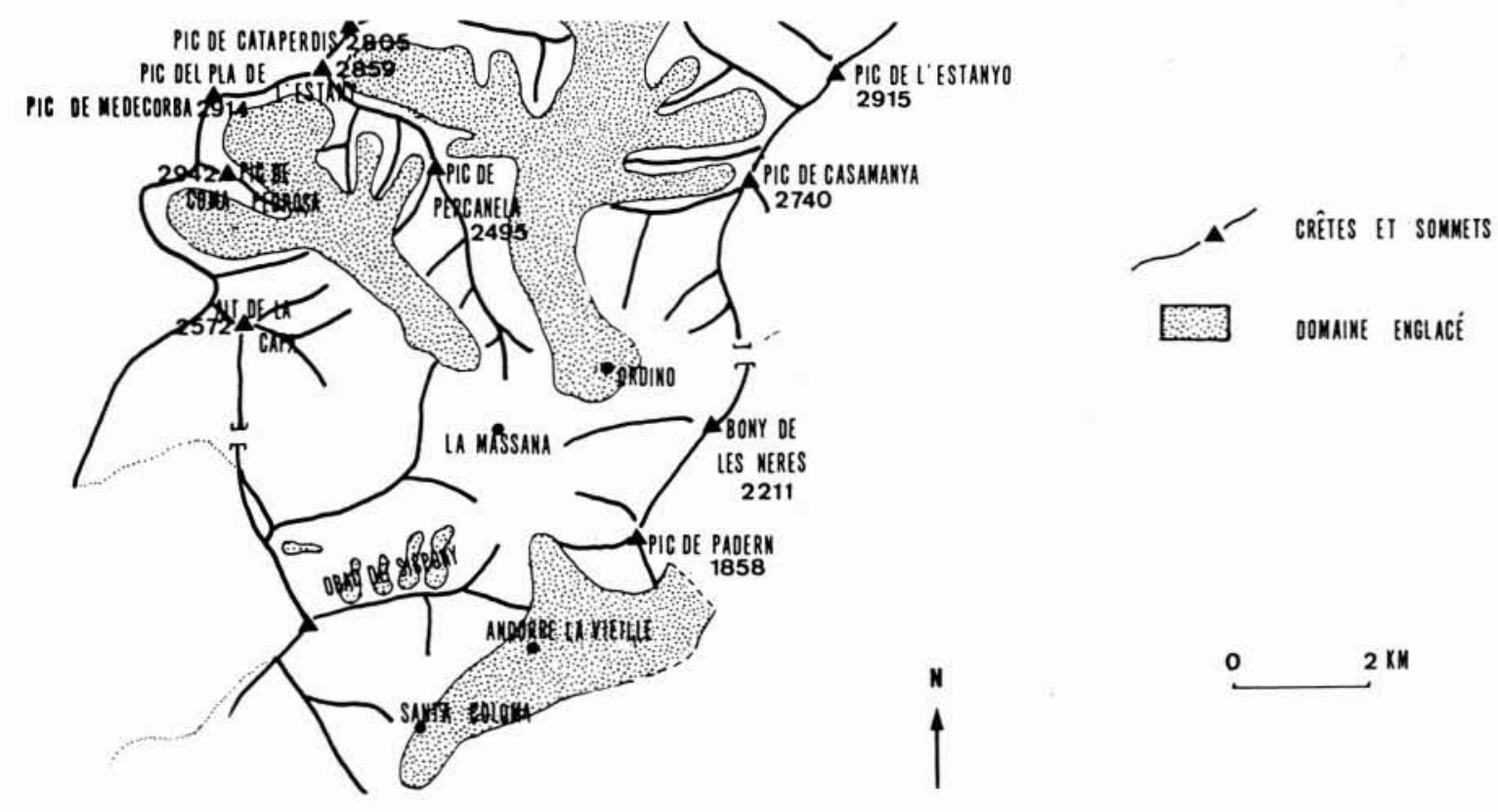

Figure 6 - L'englacement de l'ouest de l'Andorre lors de la phase de stationnement du Maximum. (d'après M.C. Prat, 1980). 
Les chiffres donnés pour le Massif du Canigou indiquent, par extrapolation, qu'une telle dynamique aurait pu se développer jusqu'à $1700 \mathrm{~m}$ en Andorre.

Les connaissances récentes sur la dynamique des glaciers rocheux tempèrent la très grande imprécision qu'il y a à transposer des limites d'un massif montagneux à l'autre et pour des époques différentes; en particulier, on peut se demander quel peut être le comportement de la bulle de pergélisol sur un versant en pente aussi forte.

\section{Les particularités de l'Obac de Sispony}

La crête de l'Obac de Sispony est la plus basse de l'Andorre à porter des marques d'érosion glaciaire. En général, les crêtes d'altitude inférieure à $2500 \mathrm{~m}$ ne portent pas une telle empreinte dans cette région. Il y a certainement des conditions climatiques qui ont favorisé l'englacement du versant. La suralimentation neigeuse par déflation sur les lambeaux de surfaces mûres, situées au vent du versant, est une de ces conditions. Le Planell de la Tossa et la Tossa Momo ont pu fonctionner en surface de déflation par vents de NW.

Dans certains cas $M$. Evin évoque, à propos de glaciers rocheux alpins, la possibilité de formation d'un béton de glace par regel nocturne des eaux provenant de la fonte diurne de la neige. Ce regel serait permis par l'effet de Balch, c'est-à-dire une circulation d'air entre les blocs qui facilite le regel. La langue rocheuse du Clot del Gel présente actuellement des conditions qui seraient favorables à un tel effet si elle était située plus en altitude, car la structure largement openwork, la rareté des fines observées même dans le talus frontal, peuvent faciliter la formation de glace de ségrégation par pénétration de la neige; Y. Veyret (1981) l'a noté pour les glaciers rocheux des Torngats.

\section{Hypothèse d'interprétation des formations à blocs du Clot del Gel}

Par le matériel le bourrelet en croissant à $1760 \mathrm{~m}$ d'altitude est différent de la langue rocheuse qui lui fait suite. La proportion importante de fines relevée par la présence de la rhodoraie, implique, selon le schéma décrit par $P$. Barrère dans les Pyrénées, qu'il s'agit d'une moraine frontale élaborée après désenglacement total du cirque. Cette période de désenglacement ayant été mise à profit par la cryoclastie pour réduire les débris à l'état de grèze. C'est ce schéma que l'on observe pour les bourrelets morainiques des stades de retrait du Post-maximum dans les massifs voisins. Physionomiquement, le bourrelet arqué du Clot del Gel ressemble aux bourrelets post-maximum que l'on peut observer au Coma Pedrosa, par leur allure très pincée et la pente externe forte.
Cette jeunesse des formes plaide pour un âge würmien et non rissien, comme l'écrit M.C. Prat. L'étude des langues glaciaires des grandes vallées d'Andorre montre qu'il n'y a jamais eu, au cours du Maximum, un désenglacement total qui aurait permis l'élaboration de grèzes.

L'attribution du bourrelet en croissant à la glaciation post-maximum pose le problème de l'altitude de la ligne d'équilibre glaciaire sur le versant de Sispony; peut être faut-il y voir l'influence des conditions mésoclimatiques précédemment décrites.

En aval du bourrelet arqué, les quatre petits arcs que dessine la langue rocheuse, semblent résulter du fluage de la base du bourrelet. Ce schéma, s'il peut être démontré, s'inscrit bien dans une hypothèse de mise en place du bourrelet au Post-maximum et d'une dynamique synchrone de fluage de la langue rocheuse.

\section{Conclusion}

Les formes et les formations à blocs de l'Obac de Sispony sont extrêmement originales dans l'Est des Pyrénées, par l'altitude à laquelle elles se trouvent. Elles diffèrent des descriptions faites jusqu'à présent à des altitudes supérieures à $2000 \mathrm{~m}$ par G. Angely (1967), F. Taillefer (1964) dans les Pyrénées ariégeoises, et G. Soutadé dans les Pyrénées orientales et qui ne concernaient que les glaciers rocheux tardiglaciaires.

La langue rocheuse du Clot del Gel, que nous serions enclins à qualifier de dépôt de glacier rocheux, est beaucoup plus ancienne.

Nous ne savons pas si ce sont les conditions particulières de l'Obac de Sispony (conditions mésoclimatiques et lithologiques, de fragilisation de la roche) qui ont permis son élaboration ou si ce sont les conditions d'englacement de la vallée du Riu Montaner qui ont permis sa conservation. Le fait que cette vallée soit préglaciaire constitue un élément remarquable et une coïncidence importante. Les moraines du Maximum n'ont pas été étalées, comme elles l'ont été ailleurs, et l'englacement post-maximum a été tout à fait insuffisant pour nettoyer le versant des héritages précédents.

Si l'Obac de Sispony présente une morphologie tellement originale, c'est parce que des héritages très anciens ont pu s'y maintenir.

Ouvrages cités

ANGELY G., 1967. - Anciens glaciers rocheux dans l'Est des Pyrénées centrales, Revue géographique des Pyrénées et du Sud-Ouest, XXXVIII, 1, p. 5-25.

EVIN M., 1983. - Structure et mouvement des glaciers rocheux des Alpes du Sud, Thèse $3^{e}$ cycle - Grenoble - $343 \mathrm{p}$.

GALIBERT G., 1965. - La haute montagne alpine, Thèse, imprimerie F. Boisseau - Toulouse.

GUITER V., 1965. - Une forme montagnarde : le rock glacier, Revue de Géographie Alpine n ${ }^{\circ} 3,60$, p. 467-487. 
PRAT M.C., 1980. - Montagnes et vallées d'Andorre. Thèse $3^{e}$ cycle, 265 p., Bordeaux.

SOUTADÉ G., 1973. - Aspects du modelé périglaciaire supraforestiers des Pyrénées Orientales, Bulletin de l'Association Française pour l'étude du Quaternaire, $\mathrm{n}^{\circ} 37,4$, p. 239-254.

SOUTADÉ G., 1980. - Modelé et dynamique actuelle des versants supra-forestiers des Pyrénées Orientales, Imprimerie Coopérative du Sud-Ouest, 452 p., Albi.

TAILLEFER F., 1968. - Le néoglaciaire pyrénéen, Mélanges de Géographie O. Tulipe, t 1, Ed. J. DUCULOT, p. 134-148, Grenoble.

VEYRET Y., 1981. - Quelques aspects de l'évolution postwisconsienne du bourrelet des Torngats, Recherches géographiques à Strasbourg, $\mathrm{n}^{\circ}$ 16-17 p. 161-167.

\section{Discussion}

Président : M. L. de CRÉCY

Le président remercie Monsieur J.PH. Desfarge et ouvre la discussion

M. Lliboutry. - Je félicite Monsieur Desfarge pour son intéressante communication et la manière dont il a étudié le glacier rocheux comme résidu d'un glacier à certaines époques. $\mathrm{La}$ problématique liée à ce type d'étude souffre de deux défauts :

1) le glacier rocheux est rattaché au permafrost (cf. travaux américains et certains travaux suisses) ce qui fausse les conclusions sur le Quaternaire.

2) On veut faire de l'étude des glaciers rocheux une discipline particulière, indûment rattachée à des questions géomorphologiques coupées de la glaciologie. Les glaciers rocheux constituent un vaste domaine d'étude. En tant que président de la Commission des neiges et glaces, je voudrais développer ces études.

Le Président. - Comment expliquez-vous cette différence d'altitude de 600 mètres entre la ligne de névé et le front du glacier rocheux, alors qu'elle est généralement de 300 mètres (Alpes) ?

M. DESFARGE. - Les limites ligne de névé-front du glacier rocheux sont sujettes à caution sur un versant aussi pentu dans l'hypothèse du déplacement d'une bulle de pergélisol. Dans le massif du Canigou, $600 \mathrm{~m}$ séparent effectivement l'atltitude de la ligne d'équilibre glaciaire du front du glacier rocheux ( $F$. TAlL. LEFER, 1968 et G. SoutAde, 1973).

$M^{m r}$ EVIN. - Le calcul de l'altitude du front des glaciers rocheux par rapport à la ligne de névé a fait l'objet de nombreuses discussions (cf. travaux de HAEBERLI).

M. Lliboutry. - Beaucoup de glaciers rocheux (Andes de Santiago) descendent au dessous de 600 mètres de la ligne de névé. La ligne d’équilibre dans ces régions n'est pas bien définie. On pourrait en discuter longtemps.

$M^{m+}$ EVIN. - ll semble difficile pour certaines personnes présentes d'admettre un écart altitudinal de 600 mètres entre la ligne d'équilibre glaciaire et le front du glacier rocheux. Il existe, au moins, deux grands types de glaciers rocheux, ceux qui fonctionnent en continu le plus souvent à l'aval des glaciers (" complexes de transition" - " ablation complexe") et ceux qui, loin de tout appareil glaciaire, sont dûs au fluage de matériel gelé. D. BARSH a démontré que les fronts de glaciers rocheux actifs se situaient aux environs de l'isotherme $-2 \circ$. Dans certains cas, une altitude plus basse encore pourrait être atteinte par des glaciers rocheux fonctionnant en un seul épisode, par départ d'une "bulle " de pergélisol dont on voit la cicatrice au lieu de départ du matériel. Ce mouvement de grande ampleur les amènerait peut-être 600 mètres au dessous de la ligne d'équilibre glaciaire s'ils sont constitués de matériel morainique. Y-a-t'il un endroit où le départ du matériel est visible?

M. Desfarge. - Il existe un creux prononcé en amont du bourrelet arqué, mais on ne sait pas s'il s'agit d'un creux de départ d'une bulle de pergélisol ou du "logement" du névé. Les extrémités de l'arc se prolongent très loin sur la pente amont sous forme de bourrelets latéraux aplatis.

M. LLIBOUtRY. - On peut observer des fluages de matériaux sur dalles polies couverts d'un sol mince (col du Lautaret) qui ne sont pas des glaciers rocheux. On trouverait plus de glaciers rocheux si l'on ne voulait pas absolument les limiter aux formations à blocs; la différenciation avec les glissements et solifluxion (sol sur niveaux imperméables) serait plus difficile. Revenons sur l'idée de bulle que je partage: ce sont de petits "surges" provoquant des avances tous les 20 ou 50 ans (cf. article sous presse dans "Satellite Image Atlas of Glaciers " et travaux avec Monsieur VALLoN et les Argentins dans le Massif de l'Aconcagua) : de petits glaciers sont en zone d'ablation peut-être pendant 30 ans avec de temps à autre des avancées brusques. Une telle avancée implique qu'il n'y a pas un état de régime à l'échelle de l'année, ni une ligne d'équilibre à tant de centaines de mètres en dessous. Selon la topographie locale, cela va plus ou moins. A certains endroits, des épaulements se forment à cause d'un freinage local. Ce n'est pas le sol qui est devenu "permafrost " et a formé une bulle de permafrost mais l'inverse. Une couche d'éboulis-mélangé de neige et de glace et de permafrost forme un terrain gelé, qui s'épaissit de telle manière que s'établit une fusion à la base. La loupe glisse alors.

A propos du problème des isothermes, je mets en garde les géographes. Les isothermes de l'air sont mal connus. Des mesures récentes, dans les Andes de Santiago, montrent que l'été, en raison de la sécheresse, le gradient moyen est de $0,9^{\circ} \mathrm{C} / 100$ mètres et non $0,7^{\circ} \mathrm{C}$ ce qui change l'altitude des isothermes. Par ailleurs, les écarts thermiques sol-air peuvent être très grands, $20^{\circ} \mathrm{C}$ ou plus. Les températures au sol n'ont rien à voir avec la température moyenne de l'air.

$M^{m r}$ EVIN. - Il s'agit de moyennes annuelles de longues périodes ( 30 à 60 ans) R.K. HANGEN et als (Fairbanks, 4th Internat. conference on permafrost, 1983) ont montré qu'il existe une relation linéaire entre le MAAT (air) et le MAGT (température du sol gelé, upper permafrost) ce qui confirme la validité de la méthode.

M. LLIBOUtRY. - Les valeurs moyennes n'ont guère de significations. Les phénomènes catastrophiques sont essentiels.

M. BEZINGE. - Jappuie la thèse de Madame Evin. Le plus bel exemple suisse se trouve dans la région de Champex dans le Massif du Trient, climat voisin de celui du Mont-Blanc. La limite d'équilibre des glaciers descend à 2800 mètres avec des altitudes de front situées à 2000 mètres. A l'occasion de travaux, une coupe a montré une glace très ancienne, un glacier noir, sous une couverture rocheuse et une forêt en versant Nord et ce à 1500 mètres d'altitude. La meilleure explication : c'est un site de couloir oủ se produit un phénomène de drainage-fusion en altitude et en face Nord avec refroidissement et regel des eaux vers le bas (cf. Tournée de Glaciologie de la Société Hydrotechnique de France en 1982).

M. SOUTADÉ. - Une des observations les plus intéressantes de M. Desfarge porte sur l'âge éventuel de ces glaciers rocheux. Jusqu'à présent, dans les Pyrénées, toutes les observations avaient rattaché ces formes à la dernière phase froide, c'est-à-dire au Tardiglaciaire. Or, il s'avère qu'ici du moins elles sont plus anciennes, au moins würmiennes.

M. le Président. - Il faudrait envisager une approche palynologique. 


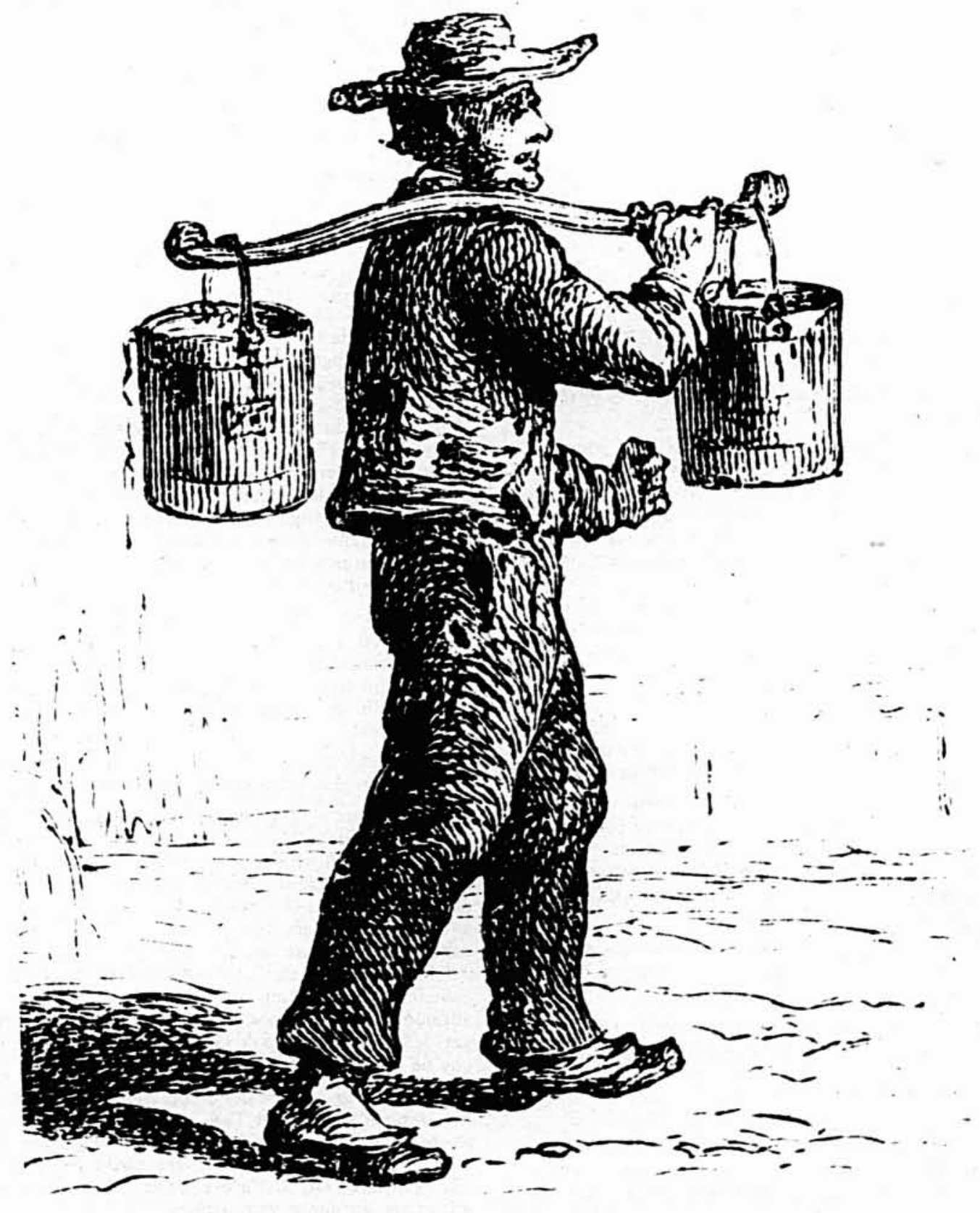

